\title{
MS20-P100 - LATE | EFFECT OF THE SIDE-CHAIN ON THE TOPOLOGY OF COORDINATION POLYMERS OF COPPER(II) WITH AMINO ACIDS
}

Smokrovic, Kristina (Department of Chemistry, Faculty of Science, University of Zagreb, Zagreb, HRV); Đilovic, Ivica (University of Zagreb, AUT); Matkovic-Calogovic, Dubravka (University of Zagreb, Faculty of Science, Department of Chemistry, Zagreb, HRV)

Over the past few decades the development of highly porous 3D coordination polymers (CP) has been of great interest, especially those derived from biodegradable and nontoxic compounds. Most of the well-known porous CPs are derived from carboxylic acids and prepared using mechanochemical or solvothermal routines.[1] That means that the structural characterization of prepared materials is usually done by X-ray powder diffraction, since growing single crystals of the right size and quality can be difficult.

Although they are easily available and provide a way of incorporating a chiral element in the structure, 3D CPs derived from amino acids are not well studied, with the exception of materials derived from aspartic acid. [1, 2] Another reason that makes amino acids attractive building blocks for the preparation of such compounds is the great variability in solubility caused by the side-chain functional groups. Because of these reasons, we have decided to research the effect of the side-chain on the topology of the CPs with copper(II) and using 4,4'-bipyridine as a bridging ligand.

Novel CPs of copper(II) and 4,4'-bipyrdine with L-alanine, L-proline, L-threonine and D- and L-valine were synthesized and structurally characterized using single crystal diffraction datasets from in-house and synchrotron instruments. Interlocked, helical cation chains are common motifs present in all of the crystal structures, with large, solvent accessible voids. All of the prepared compounds are isostructural, except for polymers derived from L-proline. Amino-acids with large side-chains produce 2D CPs with a distorted honeycomb topology, without the large solvent accessible voids.

Acknowledgments:

This work has been funded by the Croatian Science Foundation (project no. IP-2014-09-4274). The experimental data was collected at Elletra Sincrotrone Trieste.

[1] Barrio, J. P., Rebilly, J., Carter, B., Bradshaw, D., Bacsa, J., Ganin, A. Y., Park, H., Trewin, A., Vaidhyanathan, R., Cooper, A. I., Warren, J. E., Rosseinsky, M. J., (2008.) Chem. Eur. J., 14, pp 4521-4532. [2] Vaidhyanathan, R., Bradshaw, D., Rebilly, N., Barrio, J. P., Gould, J. A., Berry, N. G., Rosseinsky, M. J., (2006) Angew. Chem., 118, pp 6645-6649. 